\title{
Attenuating Tumour Angiogenesis: A Preventive Role of Metformin against Breast Cancer
}

\author{
Shan Gao, Jingcheng Jiang, Pan Li, Huijuan Song, Weiwei Wang, Chen Li, and Deling Kong
}

Tianjin Key Laboratory of Biomaterial Research, Institute of Biomedical Engineering, Chinese Academy of Medical Science and Peking Union Medical College, Tianjin 300192, China

Correspondence should be addressed to Chen Li; plumlichen@163.com

Received 3 October 2014; Accepted 9 December 2014

Academic Editor: Gang Niu

Copyright (C) 2015 Shan Gao et al. This is an open access article distributed under the Creative Commons Attribution License, which permits unrestricted use, distribution, and reproduction in any medium, provided the original work is properly cited.

Metformin is one of the most widely prescribed antidiabetics for type 2 diabetes. A critical role of metformin against tumorigenesis has recently been implicated, although several studies also reported the lack of anticancer property of the antidiabetics. Given the controversies regarding the potential role of metformin against tumour progression, the effect of metformin against breast, cervical, and ovarian tumour cell lines was examined followed by in vivo assessment of metformin on tumour growth using xenograft breast cancer models. Significant inhibitory impact of metformin was observed in MCF-7, HeLa, and SKOV-3 cells, suggesting an antiproliferative property of metformin against breast, cervical, and ovarian tumour cells, respectively, with the breast tumour cells, MCF-7, being the most responsive. In vivo assessment was subsequently carried out, where mice with breast tumours were treated with metformin ( $20 \mathrm{mg} / \mathrm{kg}$ body weight) or sterile PBS solution for 15 consecutive days. No inhibition of breast tumour progression was detected. However, tumour necrosis was significantly increased in the metformin-treated group, accompanied by decreased capillary formation within the tumours. Thus, despite the lack of short-term benefit of metformin against tumour progression, a preventive role of metformin against breast cancer was implicated, which is at partially attributable to the attenuation of tumour angiogenesis.

\section{Introduction}

In recent years, epidemiological analyses have indicated a positive association between long-term diabetes and elevated risk of malignant neoplasms [1]. In particular, patients with preexisting type 2 diabetes (T2D) present a higher risk of cancer development and cancer-related mortality. Moreover, cancer patients with diabetes also showed increased mortality compared to nondiabetic cancer patients. Given the potential causal relationship between T2D and cancer, multiple plasma glucose lowering agents have been selected to be tested for potential anticancer effects, with metformin showing the most promising result.

Metformin is one of the most efficacious and safe frontline antidiabetics for type 2 diabetes (T2D). In addition to its antiglycaemic impact, recent reports also implicated critical role of metformin in tumourigenesis $[1,2]$. Indeed, antiproliferative effects of metformin have been reported in multiple tumour cell lines via several molecular pathways, including the adenosine monophosphate kinase (AMPK) pathway, the insulin receptor cascade, and the AMPKindependent RagGTPase-dependent 3mTORC1 signalling network $[1,3]$. Evidence also supports an anti-inflammatory role of metformin against cancer progression by inhibiting cancer stem cells [4]. In contrast, some studies observed no association between metformin and cancer-related mortality [5]. Results from a newly published epidemiological analysis also reported no direct association between metformin and cancer outcome [6]. Given the controversies regarding the use of metformin as potential anticancer treatment, we examined the effect of metformin against selective tumour cell lines followed by in vivo assessment of metformin on tumour growth.

\section{Methods and Materials}

2.1. Cell Culture and Viability Assay. Human breast (MCF7), ovarian (SKOV-3), and cervical (HeLa) cancer cells 


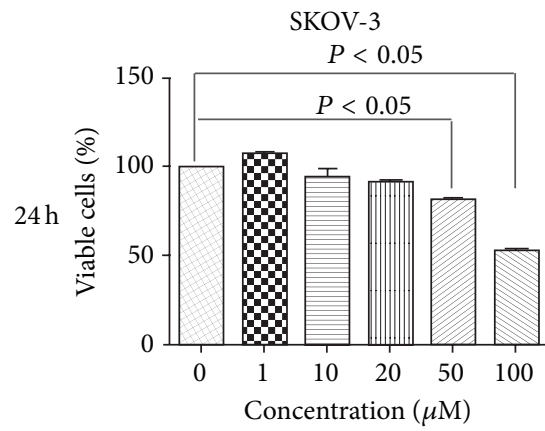

(a)

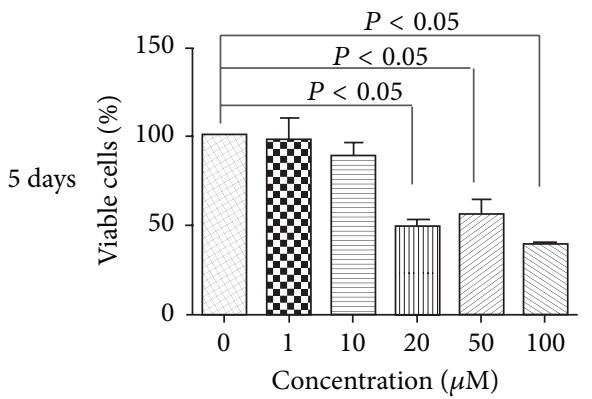

(d)

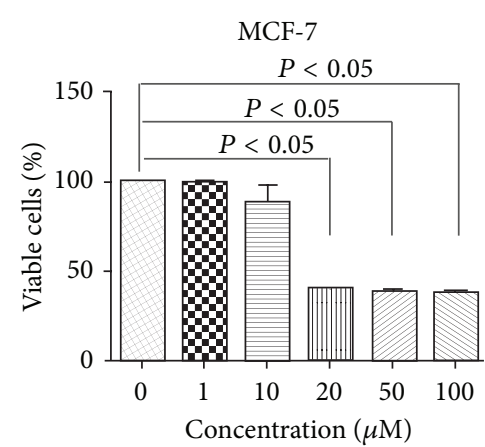

(b)

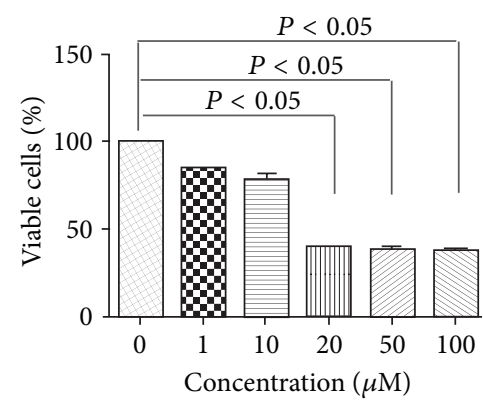

(e)

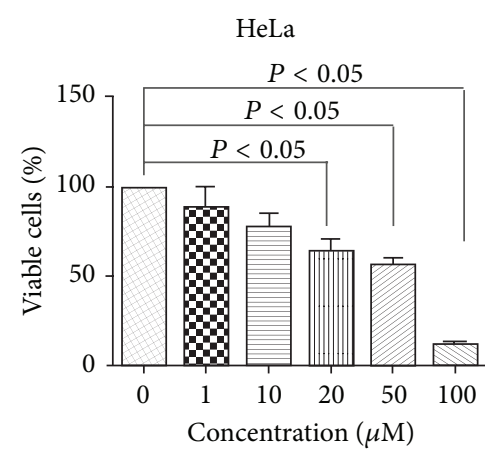

(c)

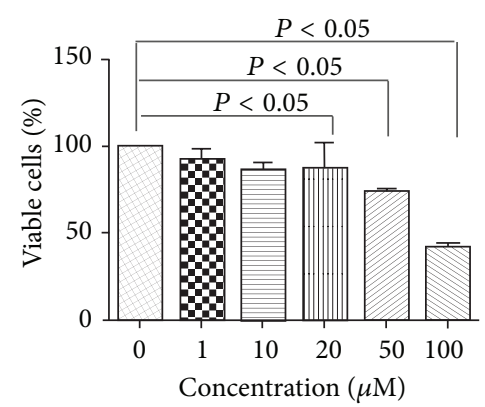

(f)

Figure 1: Metformin inhibits tumour cell growth. Human ovarian (SKOV-3), breast (MCF-7), and cervical (HeLa) cells were exposed to a series of concentrations of metformin for $24 \mathrm{~h}((\mathrm{a}),(\mathrm{b})$, and (c)) and 5 days ((d), (e), and (f)). Cell viability was assessed using a cell viability (CCK-8) assay. Data are presented as means $\pm \mathrm{SD}, n=6$.

were cultured in DMEM media (Hyclone, Beijing, China) supplemented with $10 \%$ foetal bovine serum (Gibco, Beijing, China), 100 units/mL penicillin, and $100 \mu \mathrm{g} / \mathrm{mL}$ streptomycin (Sigma-Aldrich, Beijing, China). Cells were seeded at a density of $\sim 5000$ cells per well in 96-well plates and maintained at $37^{\circ} \mathrm{C}$ under standard culturing conditions. Cells were exposed to a series of concentrations of metformin (SigmaAldrich, Beijing, China) continuously and cell viability was determined at the end of $24 \mathrm{~h}$ and 5 days using a cell counting kit-8 (CCK-8; Dojindo, Japan).

2.2. In Vivo Assessment. Xenograft breast tumour models were established by injecting MCF-7 cells into 6-week-old female BALB/c nude mice (Charles River Laboratories, Beijing, China). Once the tumour size reached $\sim 100-150 \mathrm{~mm}^{3}$, mice were randomly assigned to either control group or metformin-treated group. Local injection of metformin (20 mg/kg body weight) or sterile PBS was administered for 15 consecutive days. Changes of body weight were monitored and tumour volumes were measured and corrected according to standard formula [7].

2.3. Histomorphological and Immunofluorescence Analysis. 15 days after initial injection, tumours were dissected and fixed in $4 \%$ paraformaldehyde before being paraffin embedded. Consecutive sections (thickness, $5 \mu \mathrm{m}$ ) were cut onto microscope slides. Haematoxylin and eosin (H\&E) staining was employed to examine tumour morphology and immunofluorescent staining using an antibody raised against von Willebrand factor (vWF; 1:200 dilution; Dako, Shanghai, China) was also carried out to evaluate capillary formation. The staining data were analysed with a fluorescent microscope (Leica, Germany) and fluorescent intensity was quantified using ImageJ software (National Institute of Health, USA).

\section{Results}

3.1. Metformin Inhibits In Vitro Tumour Cell Growth. Given the high prevalence of ovarian, cervical, and, particularly, breast cancers in pre- and postmenopausal women, 3 female tumour cell lines, MCF-7, SKOV-3, and HeLa, were initially selected to investigate the potential anticancer effect of metformin in vitro. As shown in Figures 1(b) and 1(c), $24 \mathrm{~h}$ exposure to metformin significantly reduced cell viability in all 3 tumour cell lines, with a maximum response of $42 \pm$ $8 \%, 38 \pm 2 \%, 14 \pm 2 \%$ for SKOV-3, MCF-7, and HeLa cells, respectively (Figures 1(a)-1(c)). Similar inhibitory responses were also observed from cells treated with metformin for 5 days (Figures 1(d)-1(f)). For both SKOV-3 and HeLa cells, the metformin-exerted attenuation of cell growth appeared to be concentration-dependent, in contrast to MCF-7, of which the inhibitory responses were similar once the administrative dose of metformin was over $20 \mathrm{mM}$. However, as noted by the National Cancer Institute some years ago, the activity of a pharmacological agent in vitro does not necessarily reflect its 


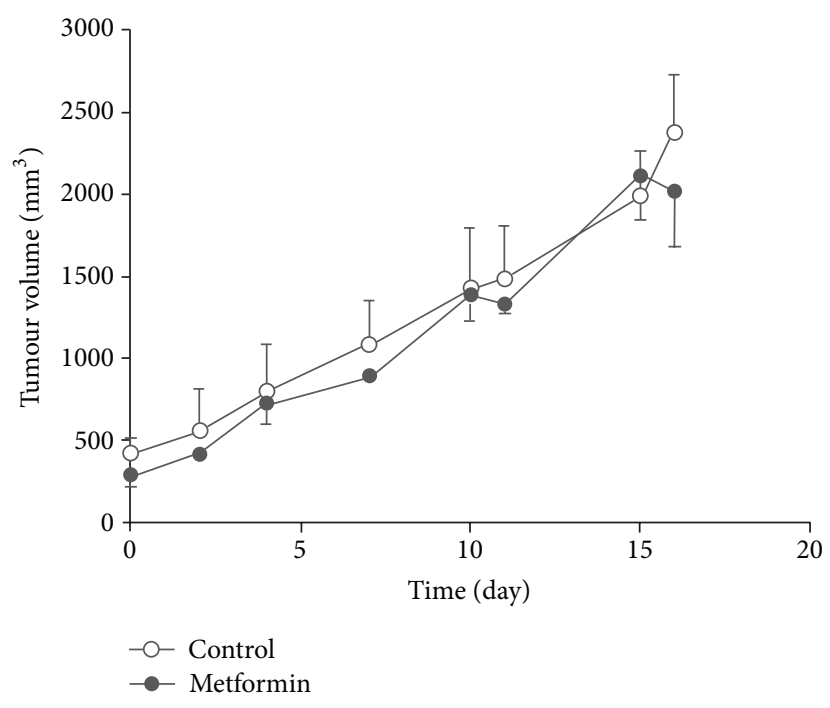

(a)

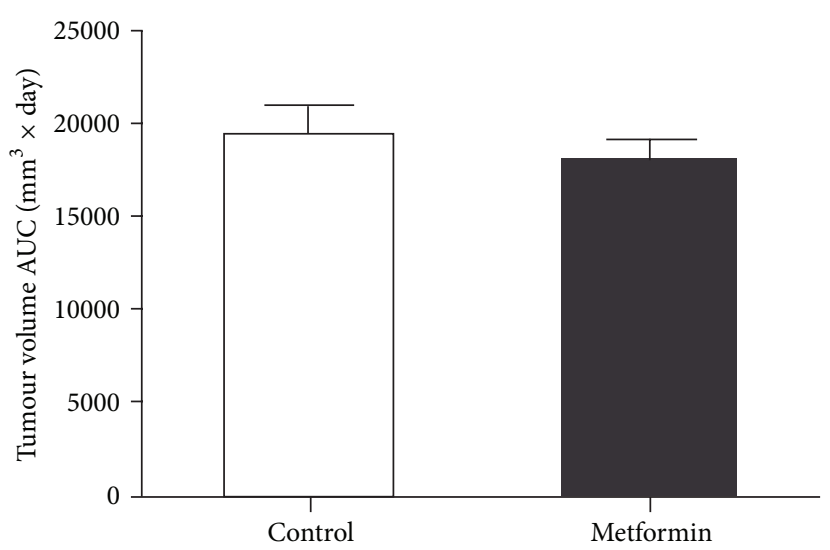

(c)

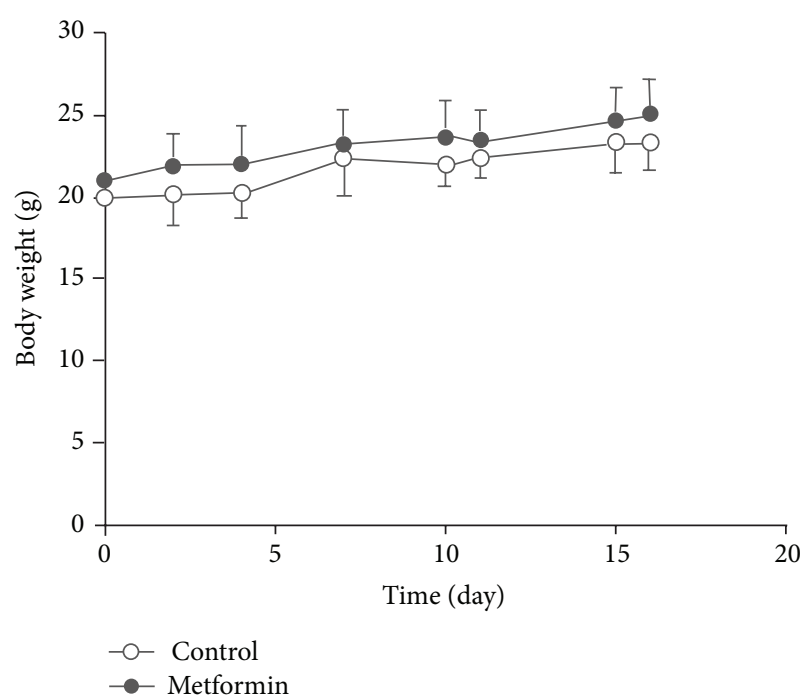

(b)

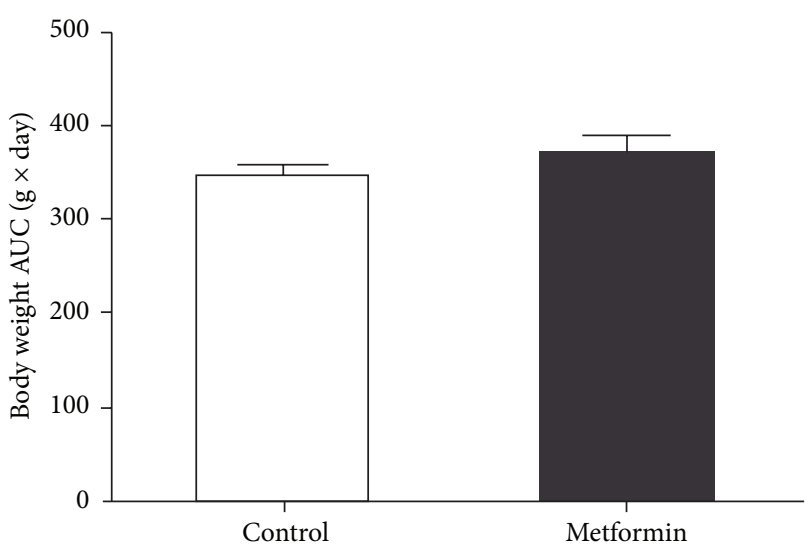

(d)

Figure 2: In vivo assessment of metformin treatment on tumour growth. Metformin $(20 \mathrm{mg} / \mathrm{kg}$ body weight; Metformin group) or sterile PBS (Control group) was injected locally to mice with breast carcinoma for 15 consecutive days. (a) Average tumour size and (b) body weight were monitored and plotted against time for Metformin (closed circle) and Control group (open circle). Total changes of tumour volume (c) and body weight (d) were also presented as area under curve (AUC). Data are presented as means \pm SD, $n=4-5$.

in vivo performance [8], and subsequent in vivo assessment was carried out.

\subsection{Effect of Metformin on In Vivo Tumour Progression} and Tumour Angiogenesis. Our in vitro cytotoxicity assay demonstrated marked inhibitory impact of metformin on ovarian, breast, and cervical cancer cell lines, with breast tumour cells, MCF-7, being the most responsive. Indeed, several studies have implicated a positive correlation of shortterm use of metformin and breast carcinoma remission [911]. A clinical trial study also demonstrated anticancer impact of metformin in nondiabetic postmenopausal women with estrogen receptor positive breast tumours [12]. In contrast, another report observed no inhibitory benefit of metformin on multiple subtypes of breast tumours under euglycaemic condition [13], which was further supported by epidemiological studies also demonstrating a lack of anticancer property of metformin against breast carcinoma [14].
Given the high prevalence of breast cancer and the current controversies concerning the exact impact of metformin use against breast carcinoma, human xenograft breast tumour mouse models were used in the present study for in vivo evaluation. Thus, local injection of PBS (Control group) or metformin ( $20 \mathrm{mg} / \mathrm{kg}$ body weight; Metformin group) was administered daily at the tumour site for two weeks. No changes of tumour volume were detectable between the control and metformin-treated groups (Figures 2(a) and 2(b)). No attenuation of tumour progression was observed either as superimposable tumour growth profiles were obtained from both groups (Figure 2(a)). In addition, no significant difference in animal body weight was detected between the two groups (Figure 2(b))

Despite the lack of inhibitory impact of metformin on tumour growth, subsequent histological analyses revealed marked increase of tumour necrosis in metformin-treated mice $(143 \pm 11 \%$ over control group, $P<0.01$; Figures 

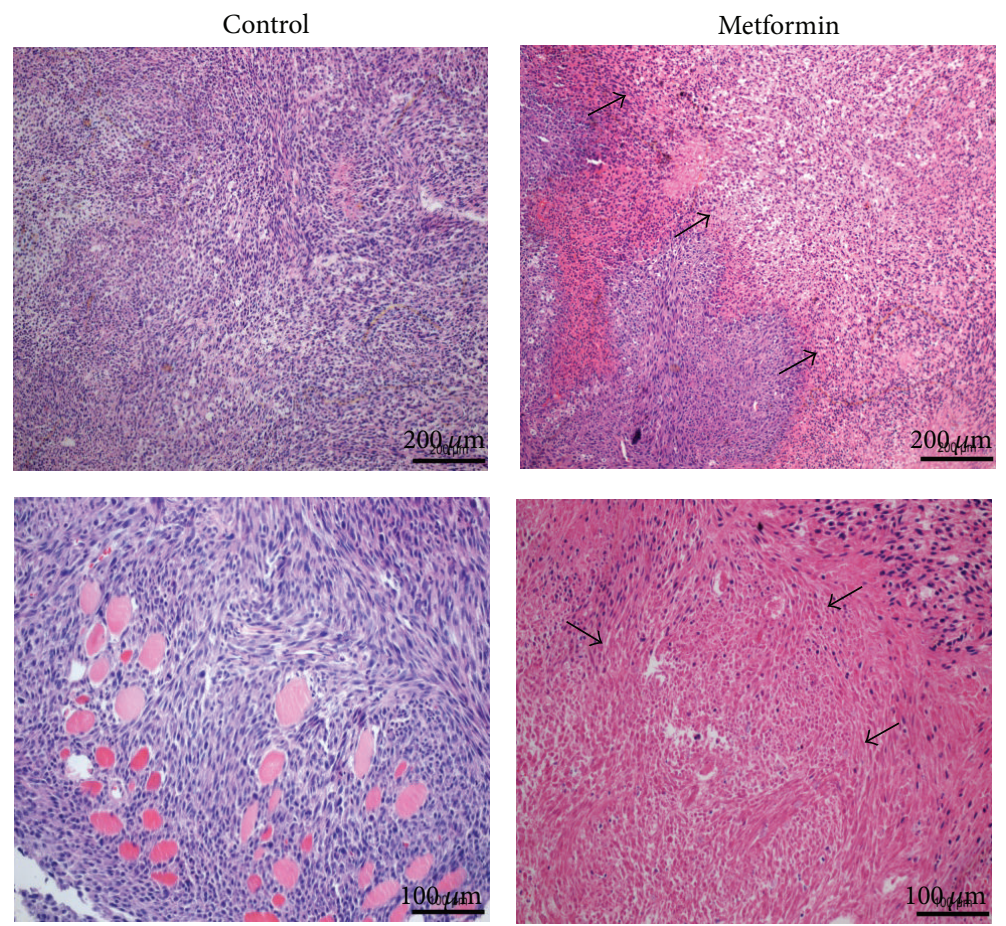

(a)
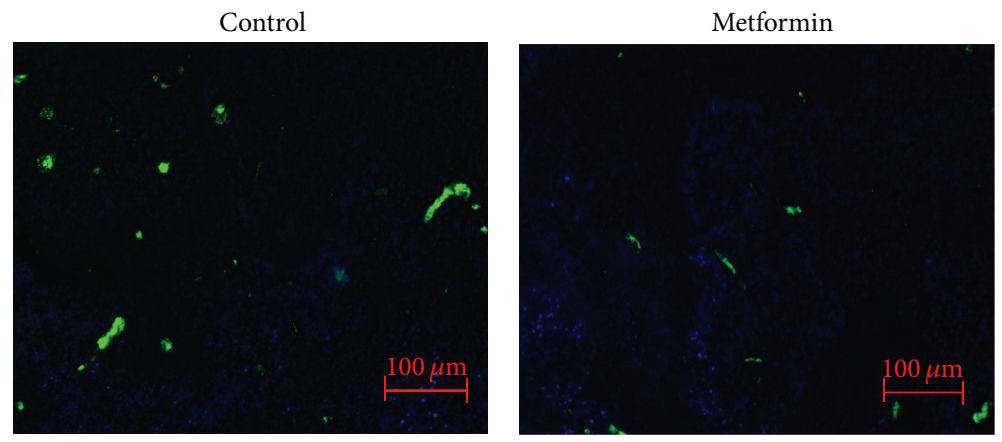

(b)

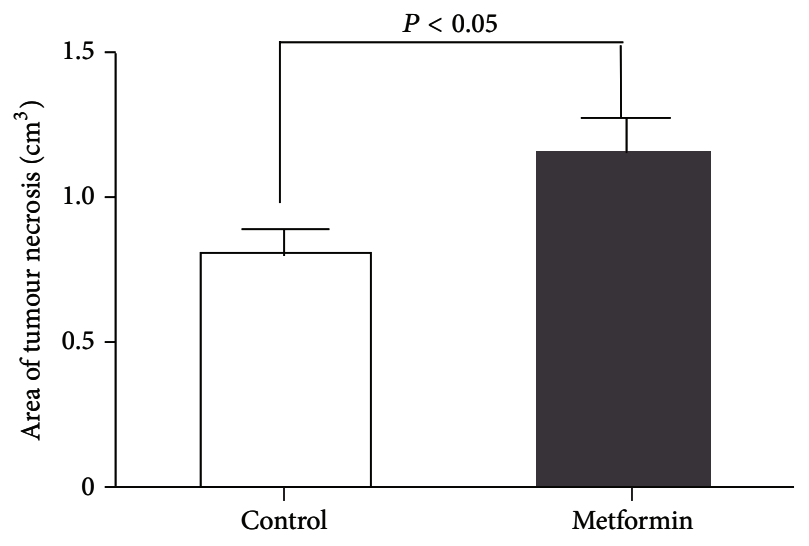

(c)

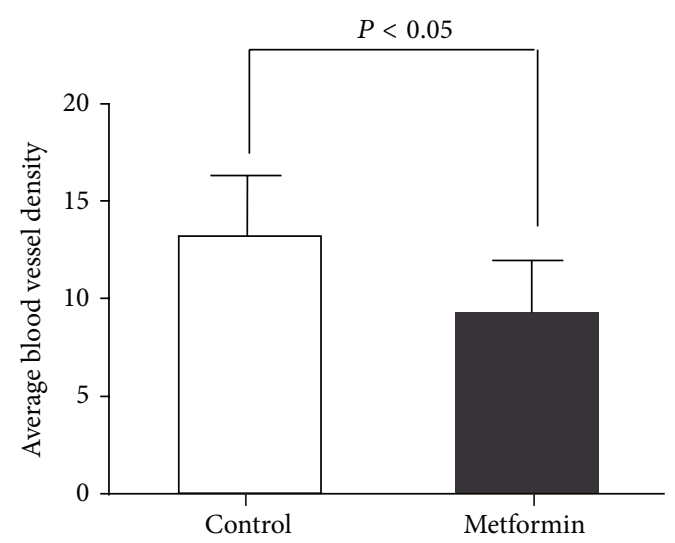

(d)

FIGURE 3: Effect of metformin treatment on tumour necrosis and angiogenesis. H\&E staining of tumours obtained from Metformin and Control groups. Scale bar: $200 \mu \mathrm{m}$ ((a), upper panels) and $100 \mu \mathrm{m}$ ((a), lower panels). (b) Immunofluorescent staining of vWF (green) in tumours obtained from Metformin and Control groups. Scale bar: $100 \mu \mathrm{m}$. Nuclei were counter-stained with DAPI (blue). Area of tumour necrosis (c) and tumour blood vessel density $(\mathrm{d})$ were quantified. Tumour necrosis was indicated by black arrows. Data are presented as means $\pm \mathrm{SD}, n=4-5$. Images are representative of 4-5 animals from 3 separate experiments. 
3(a) and 3(c)). Furthermore, immunofluorescence staining of von Willebrand factor (vWF), a microvascular endothelial marker, also revealed reduced average blood vessel density in tumours obtained from the metformin-treated animals (69 $\pm 29 \%$ over control group, $P<0.02$; Figures 3 (b) and 3(d)), implicating an antiangiogenic impact of metformin.

\section{Discussion}

The cytotoxicity of metformin was observed in all 3 cell types, with breast tumour cells being the most responsive, although cautions need to be exercised when drawing conclusions from in vitro results since cultured tumour cells are morphologically and functionally different from native tumours.

Subsequent in vivo assessment showed no detectable tumour reduction after local injection of metformin $(20 \mathrm{mg} / \mathrm{kg}$ body weight) for 15 days. In fact, the effect of metformin against breast cancer has long been extensively investigated albeit contradictory as summarized in a recent review [2]. Most studies have reported decreased incidents and severity of mammary cancer in rodent models after long-term oral or intravenous administration of metformin. Similarly, attenuated tumour progression was observed in humans following treatment with high dosage metformin [2]. In contrast, no inhibition of tumour growth and latency was also recorded, often when low dosage of metformin was applied. Considering the potential implications of different dosage and administrative routes of metformin treatment on cancer outcome, $20 \mathrm{mg}$ per $\mathrm{kg}$ body weight metformin was used in the present study and the drug was directly injected to the tumour sites to minimize non-tumour-site distribution caused by different routes of administration [2]. We observed no attenuation of tumour growth after shortterm administration of a moderate level of metformin, which suggests limited short-term anticancer ability of metformin treatment per se. However, this result may not reflect longterm effect of the drug as the necrosis area was considerably larger in tumours obtained from metformin-treated mice.

In addition, significant attenuation of capillary formation was also evident from the metformin-treated group, consistent with a previous report proposing an AMPK/mTORdependent antiangiogenic effect of metformin on ovarian cancer [15]. Thus, despite the lack of short-term benefit of metformin in tumour regression in vivo, a preventive role of metformin against breast cancer was implicated, which is at least partially attributable to the attenuation of tumour angiogenesis. Further investigation is required to evaluate whether the antiangiogenic effect of metformin is tumourspecific, particularly since metformin is widely prescribed as an antidiabetic and T2D patients have an elevated risk of vascular disorders. Furthermore, considering the diversity of metformin action, the exact mechanisms underlying the antiangiogenic property of metformin are also required to be elucidated.

\section{Conflict of Interests}

The authors declare that there is no conflict of interests.

\section{Acknowledgments}

This study was financially supported by the National Natural Science Foundation of China (nos. 31300732 and 81301309) and the Tianjin Research Programme of Application Foundation and Advanced Technology (no. 13JCYBJC39300).

\section{References}

[1] C. Li and D. Kong, "Cancer risks from diabetes therapies: evaluating the evidence," Pharmacology \& Therapeutics, vol. 144, no. 1, pp. 71-81, 2014.

[2] V. N. Anisimov, "Do metformin a real anticarcinogen? A critical reappraisal of experimental data," Annals of Translational Medicine, vol. 2, no. 6, p. 60, 2014.

[3] A. Kalender, A. Selvaraj, S. Y. Kim et al., "Metformin, independent of AMPK, inhibits mTORC1 in a rag GTPase-dependent manner," Cell Metabolism, vol. 11, no. 5, pp. 390-401, 2010.

[4] H. A. Hirsch, D. Iliopoulos, and K. Struhl, "Metformin inhibits the inflammatory response associated with cellular transformation and cancer stem cell growth," Proceedings of the National Academy of Sciences of the United States of America, vol. 110, no. 3, pp. 972-977, 2013.

[5] X. Sui, Y. Xu, J. Yang et al., "Use of metformin alone is not associated with survival outcomes of colorectal cancer cell but AMPK activator AICAR sensitizes anticancer effect of 5fluorouracil through AMPK activation," PLoS ONE, vol. 9, no. 5, Article ID e97781, 2014.

[6] R. J. Stevens, R. Ali, C. R. Bankhead et al., "Cancer outcomes and all-cause mortality in adults allocated to metformin: systematic review and collaborative meta-analysis of randomised clinical trials," Diabetologia, vol. 55, no. 10, pp. 2593-2603, 2012.

[7] W. Wang, C. Li, J. Zhang, A. Dong, and D. Kong, "Tailor-made gemcitabine prodrug nanoparticles from well-defined drugpolymer amphiphiles prepared by controlled living radical polymerization for cancer chemotherapy," Journal of Materials Chemistry B, vol. 2, no. 13, pp. 1891-1901, 2014.

[8] C. L. Morton and P. J. Houghton, "Establishment of human tumor xenografts in immunodeficient mice," Nature Protocols, vol. 2, no. 2, pp. 247-250, 2007.

[9] P. J. Goodwin, V. Stambolic, J. Lemieux et al., "Evaluation of metformin in early breast cancer: a modification of the traditional paradigm for clinical testing of anti-cancer agents," Breast Cancer Research and Treatment, vol. 126, no. 1, pp. 215220, 2011.

[10] S. Niraula, R. J. O. Dowling, M. Ennis et al., "Metformin in early breast cancer: a prospective window of opportunity neoadjuvant study," Breast Cancer Research and Treatment, vol. 135, no. 3, pp. 821-830, 2012.

[11] S. Hadad, T. Iwamoto, L. Jordan et al., "Evidence for biological effects of metformin in operable breast cancer: aA preoperative, window-of-opportunity, randomized trial," Breast Cancer Research and Treatment, vol. 128, no. 3, pp. 783-794, 2011.

[12] J. Kim, W. Lim, E.-K. Kim et al., "Phase II randomized trial of neoadjuvant metformin plus letrozole versus placebo plus letrozole for estrogen receptor positive postmenopausal breast cancer (METEOR)," BMC Cancer, vol. 14, no. 1, article 170, 2014.

[13] S. Sadighi, S. Amanpour, B. Behrouzi, Z. Khorgami, and S. Muhammadnejad, "Lack of metformin effects on different molecular subtypes of breast cancer under normoglycemic 
conditions: an in vitro study," Asian Pacific Journal of Cancer Prevention, vol. 15, no. 5, pp. 2287-2290, 2014.

[14] N. Besic, N. Satej, I. Ratosa et al., "Long-term use of metformin and the molecular subtype in invasive breast carcinoma patients-a retrospective study of clinical and tumor characteristics," BMC Cancer, vol. 14, no. 1, article 298, 2014.

[15] R. Rattan, R. P. Graham, J. L. Maguire, S. Giri, and V. Shridhar, "Metformin suppresses ovarian cancer growth and metastasis with enhancement of cisplatin cytotoxicity in vivo," Neoplasia, vol. 13, no. 5, pp. 483-491, 2011. 


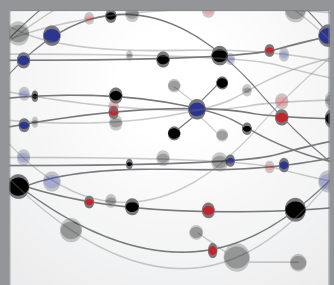

The Scientific World Journal
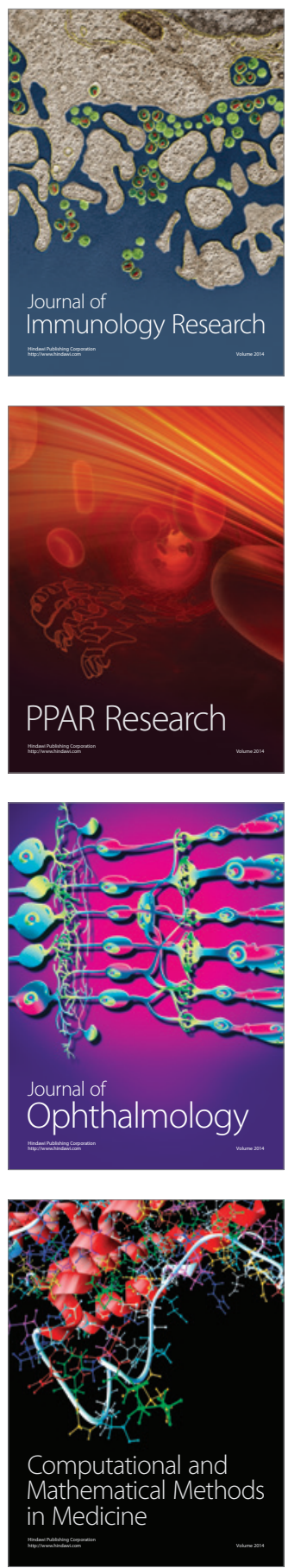

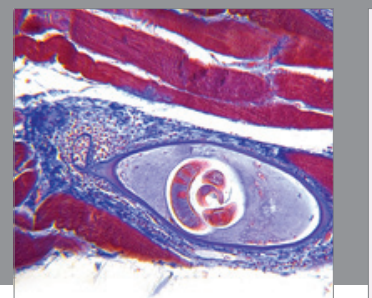

Gastroenterology

Research and Practice
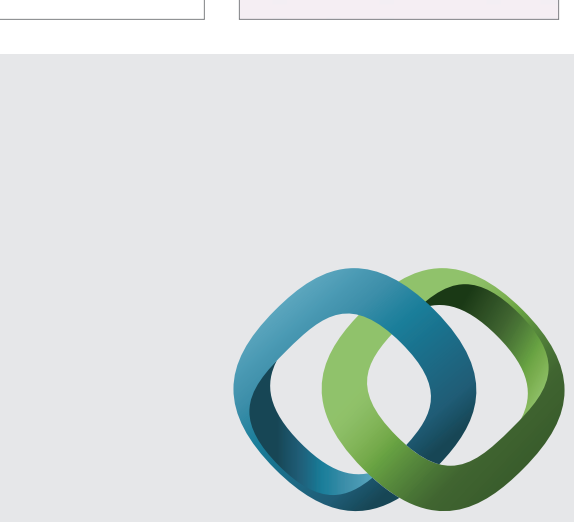

\section{Hindawi}

Submit your manuscripts at

http://www.hindawi.com
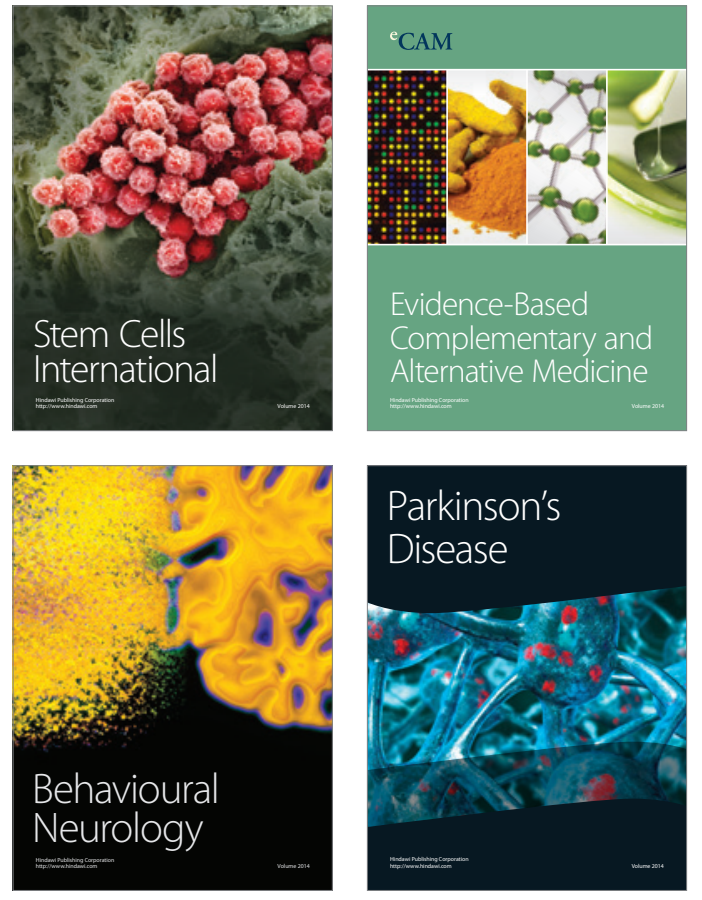
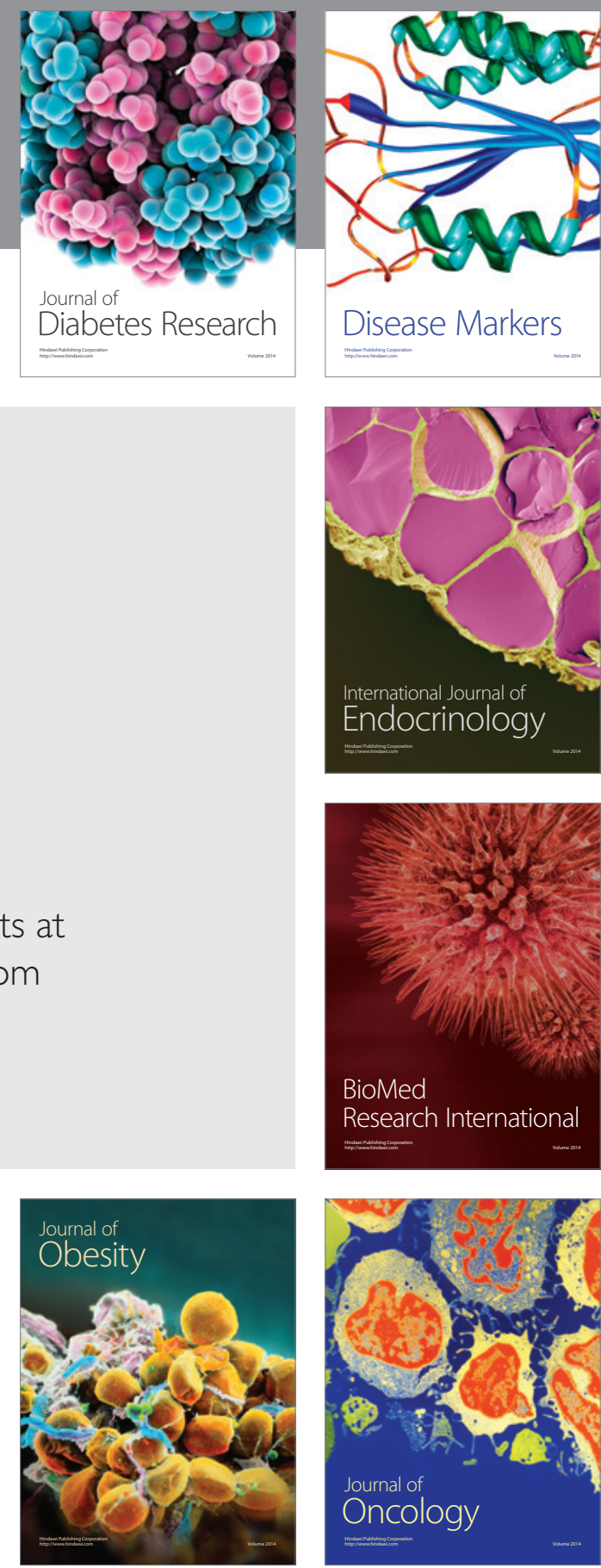

Disease Markers
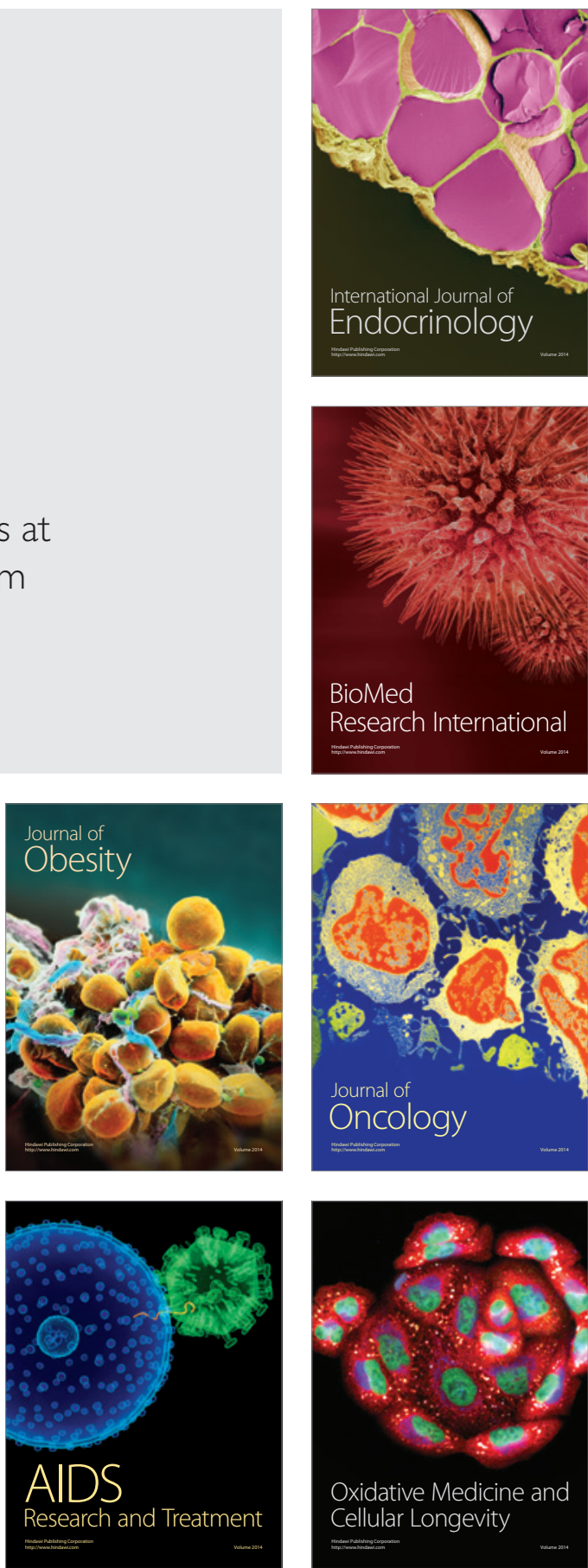